\title{
Applications of Extendable Embedded Web Servers in Medical Diagnosing
}

\author{
Chiranji Lal Chowdhary \\ School of Info. Tech. and Engg. \\ VIT University, Vellore, India.
}

\author{
Shynu P.G. \\ School of Info. Tech. and Engg. \\ VIT University, Vellore, India.
}

\begin{abstract}
An embedded web server, in general, controls the use of system resources by running the web server within tightly controlled limits, so that bugs will not compromise the system operations. In this paper we discuss on such an embedded web server which is to be secured from the root, by the use of a portable-runtime layer. Such a layer permits the portability of maximum codes. This portable-runtime layer is also called cross-platform layer. The embedded web server has also been hardened against several common DoS attacks. In the proposed embedded web server, the memory has been configured to a predefined limit, so that it rejects very large requests or URLs that are too long. The application program sends the result as web pages, using common gateway interface (CGI) protocol. This system was tested successfully on Linux / Fedora to carry out the diagnosis of the cardiac arrest.
\end{abstract}

\section{Keywords}

common gateway interface , embedded web server, HTTP, URL, denial of services.

\section{INTRODUCTION}

Recent advances in system-on-silicon technology and the miniaturization of computers and electronic devices promise embedded systems of ever-shrinking size. Such web systems are offering a promise to commercial business, education, consumer products engineering and science [17]. These systems help users to monitor and control the instruments on active Web pages.

An enterprise web server, like Apache, is having different set of design goals. The design goals and the processing architectures are different for the enterprise and embedded web servers respond and receive standard HTTP protocols. As an enterprise web servers can run over a dedicated system only. So for an enterprise server it is acceptable to scale to consume available resources and also to serve incoming requests. But compare to enterprise web server the embedded web servers are secondary to the essential functions an application or device must perform. So that the embedded web servers compulsorily minimize resource demands with deterministic in the load they place on a system $[1,17,16]$.

This study on embedded web server is based on Portable Runtime Layer that is secure from the foundation. This layer allows most of the code to be portable and also called a crossplatform layer. This embedded server gives a buffer handling module to eliminate buffer overflows. The extended embedded web servers' controls use of system resources and that is done by running the web server in close control limits that bugs will not compromise system operation $[1,17,4]$. The embedded web server was hardened with several common denials-of-service attacks. This server has been configured to pre-allocate memory and not to grow beyond predefined memory limits. It is configured to reject requests that are too large and also designed to reject URLs that are too large. The application program sends the result as web pages using common gateway Interface (CGI) protocol. The proposed embedded web server can be run successfully on Red Hat Enterprise Linux 4.0, Fedora Core 5 to carry out the diagnosis of the cardiac arrest.

The paper is organized as follows: section 2 gives a survey of available web servers; section 3 gives embedded web server; section 4 is design and implementation of embedded web server. Section 5 will be for conclusion. At the last, i.e. section 6 , references are included in the paper.

\section{LITERATURE SURVEY ON WEB SERVERS}

Now-a-days a great progress is found in the VLSI, embedded systems, and internet applications. More than 50 web servers are present in working conditions and out of that some are embedded web servers, like, RomPager from Allegro Software, Emweb from Agranat Systems, MicroServer from SpyGlass, WebPort from InterNiche, and Appweb from Embedded System Group.

An open and economical alternative to networking devices was provided by some researchers for the web servers [3]. During their work they suffer from communication reliability and efficiency in real-time. Some other researchers [2] found a possibility of modifying an existing embedded web server which could be used for ABB controllers. They tell that the web server so created is able to present a number of controller related information in dynamic web pages [2].

The work on telemedicine for homecare, describe a system where the patient at home is provided medical advice through a low cost system with PC, TV and camera [1, 17]. This work was having some limitations that the system can't electronically transmit the vital sign readings from the patient residence [19]. The nursing staff from the hospital / doctor completely relies on the patient to find or measure and read out the instrument displays. Therefore patients with limited eyesight or mobility cannot be treated through this system [19]. Their work concentrates mainly on general medicine. It is observed that at the Hays Medical Center in Kansas , the cost per visit by a registered nurse was $\$ 135$ for a physical 
visit, versus $\$ 36$ for tele-visit [17]. So if that system is implemented in that health centre a definite reduction in the cost. From the total number of the physical visits at least one third can be converted to tele-visits by the author's opinion. They claim that images taken during tele-visits also helped nurses to monitor the progress of a wound over a certain period of time [17].

The basic design concept for mini embedded web servers introduced that serves the common devices interfaces and gains a good performance by an internet [13]. This paper was efficient to be used in industry, medical, and other fields at a broad scale and also set the goal of the embedded web server to link with device, many home appliances and also, the small size of this is useful to be embedded into small appliances [13]. References [14] is providing about attaching small devices to the Internet by hardware and protocol tools as home appliances and sensors. Here main focus is on implementation of the functionally minimized web server on silicon. So hardware-based approaches are developed that is a WebChip family of IPv6-compatible solutions and this gives the feeling of embedded and minimized web systems [14].

According to one paper [4] it is said that the web access is enabled by the server for distributing the measurement or control of systems. This provides a scalable networking solution that is optimized for the instrumentation, educational laboratories, industrial and home automation. The author [4] also says that the users can monitor and control transducers on active Web pages enhanced with JavaScript. Bill Columbia [5] presents the basic concepts on the use of embedded web servers with simple applications. The author put forth the idea of using embedded web servers for time critical and non-time critical applications. Agranat Systems, Emweb [9], AllegroSoft, RomPager [10], SpyGlass, MicroServer [12] are different ideas related to the commercial use embedded web server. RomPager Embedded Web Server [18] was a HTTP 1.0/1.1 compliant web server in CGI style user exit support and having file system support. It uses from $7 \mathrm{~KB}$ to $12 \mathrm{~KB}$ of ROM code and provides a small, powerful server for low-end devices [18].

Recently published papers deal with using embedded web server for control purposes and a few published works on using embedded web server [16] for medical diagnosing applications. We worked on use of embedded server in medical diagnosing.

\section{EMBEDDED WEB SERVER}

Our objective here to work of the wireless applications, embedded systems and VLSI in the area of medical. By this paper we are trying to modify an open source embedded web server. Our main interest is in medical diagnosis of cardiac arrest. The core server is designed to use minimum system resources with high performance and minimal footprint. The core layer is the portable runtime layer. It isolates platform dependencies. The embedded web server has modular architecture with dynamic module loading. This dynamic module capability of the portable runtime layer helps the embedded web server to provide a suite of loadable handlers that serves the content for clients. This embedded web server runs on Unix/Linux platform. The proposed embedded web server continuously monitors the symptoms of the cardiac arrest. Under normal conditions of the person, the embedded web server sends a welcome page to the browser. If the embedded web server recognizes any of the symptoms mentioned in the application program, it sends the alert page to the browser.

\section{DESIGN AND IMPLEMENTATION OF EXTENDABLE EMBEDDED WEB SERVER}

The core HTTP server is kept small compared to the dynamic modules that run at the top of it. The core HTTP server is designed to provide the main HTTP processing and socket communications, initialization and parsing the configuration file and buffering. This portable runtime layer isolates platform dependencies. The embedded web server has a modular architecture where the components can be dynamically loaded at runtime.

The components of the embedded web server include server library, client library, server main program and the loadable modules for common gateway interface. The embedded web server needs $400 \mathrm{~KB}$ RAM memory, $600 \mathrm{~KB}$ ROM space, TCP / IP network stack.

The core HTTP server cannot serve any pages or documents by itself. It uses modules and handlers to serve HTTP requests. The three handlers namely static handler, CGI handler and copy handler created in the embedded web server is used for diagnosis of cardiac arrest.

The embedded web server is build from source code on $15 \mathrm{MB}$ disk space. The medical diagnosing application is developed in $4 \mathrm{MB}$ disk space. This embedded web server is highly portable so that it can be moved from one project to another project.

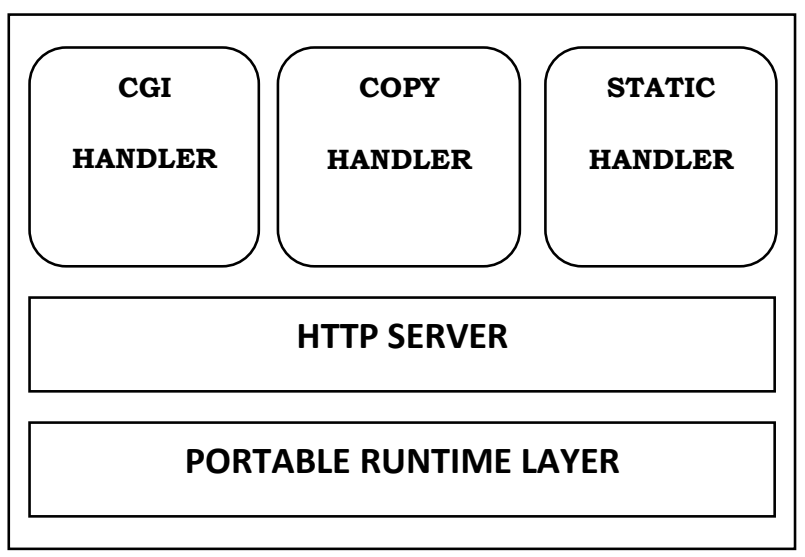

Fig. 1. Embedded Web Server System.

The embedded web server provides detailed feedback about the access to and operation of the server through the two log files error $\log$ and access $\log$. The error $\log$ records the essential configuration, denial of requests and trace information. The location of the error log file is specified via the error log configuration file and the log level directive specifies the level of logging. The log level is set to 2 for this embedded web server. The access log records the details of each successful request served by the embedded web server. 


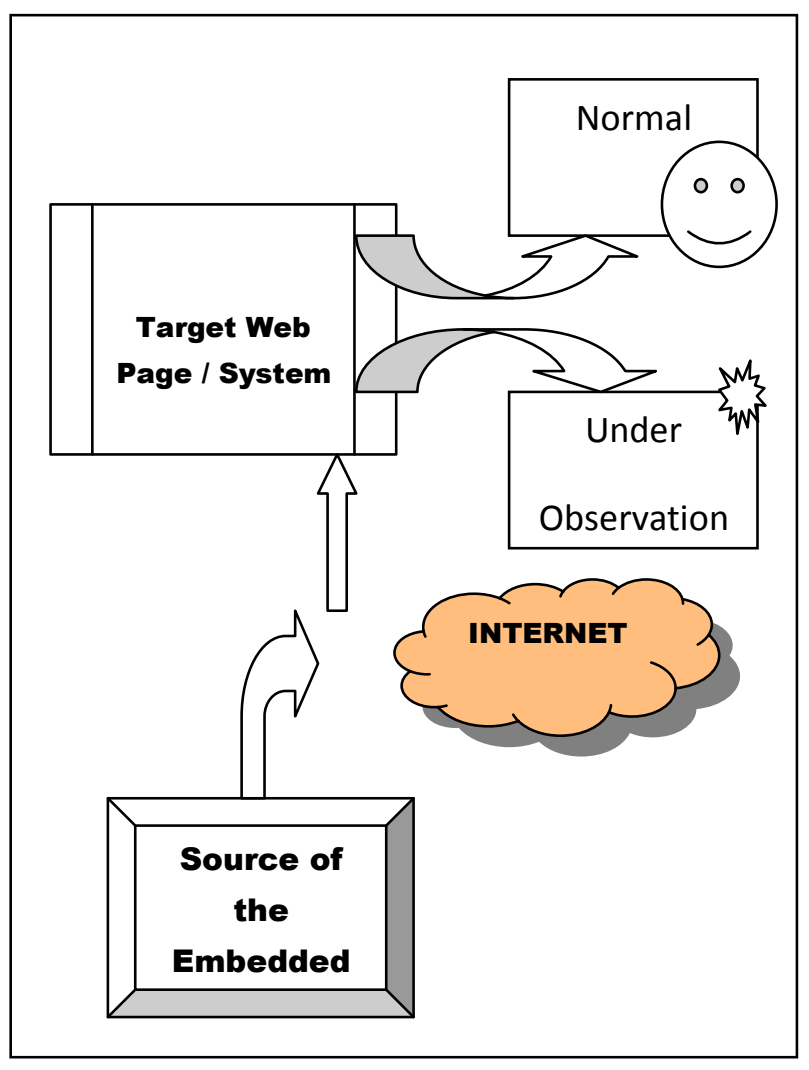

Fig 2. Simple Designed System.

When the embedded web server starts up, the configuration file is read. It controls the embedded web server configuration including the ports and addresses, the modules to be loaded and the web pages log requests. The embedded web server configuration file is processed in a single-pass.

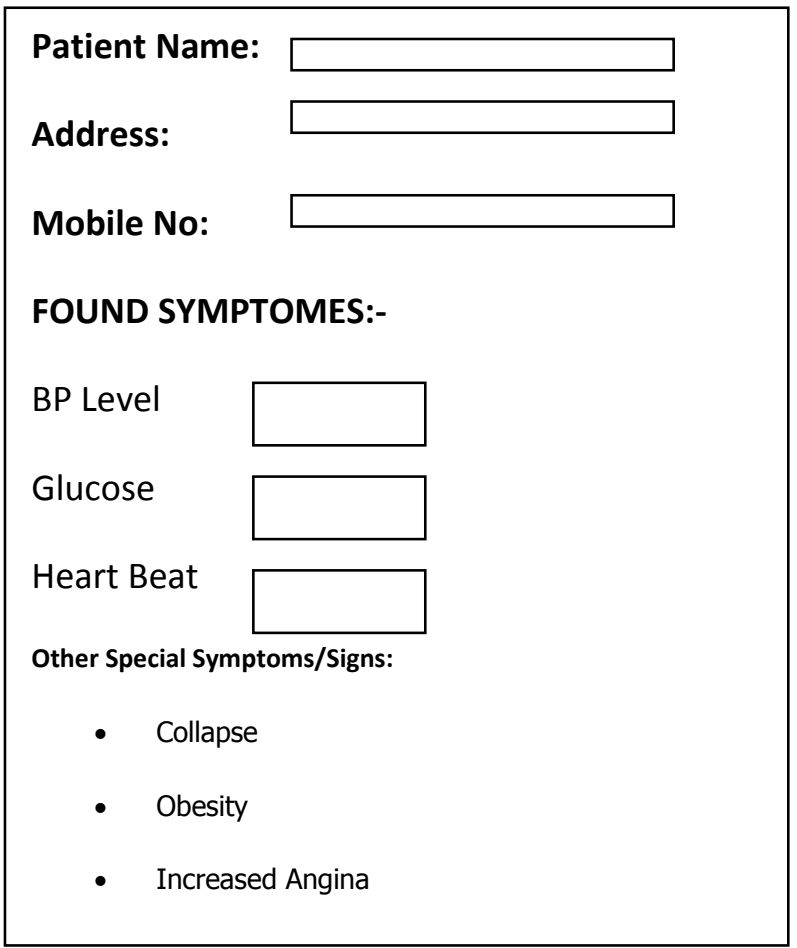

Fig 3. Patients Readings of heart for web page access.
The configuration file has to be run to generate the config.make, config.sh and config.h master build files. The configure program is a script that creates the embedded web server configuration files and it controls the build settings. The portable runtime layer, HTTP and AppWeb directories help to shake out the bugs. The HTTP web server modules are then compiled.

CGI programs are configured and invoked by URL prefix and by URL extension. ScriptAlias configuration file is the CGIbin directory. The CGI technology along with perl programming is used to generate dynamic web pages during the diagnosis of cardiac arrest.

The Assess Condition consists of a set of screen, which helps to assess the person's present condition. The web page in fig.3 is used to capture vital signs of cardiac arrest during the assessing condition. The embedded web server processes these signs with the help of the application program developed in Perl. The results are displayed as web pages in standard web browsers. These results are shown in fig 4 and fig.5.

\section{Personal Status / Condition of the}

\section{Patient:}

Mr. XYZ is in Normal Conditions.

His parameters are:

\section{Blood Pressure: $120 / 80 \mathrm{mmHg}$}

2. Glucose (F) : $90 \mathrm{mg} / \mathrm{dl}$

3.

4.

His next reading is to be taken on:

Fig 4. Personal readings of a patient in normal condition.

Fig.4. is the web page displayed by the embedded web server under the normal conditions which is continuously monitoring the application program.

Personal Status / Condition of the Patient:

WARNING!!!

ALERT!!! - Nursing staff and doctors!

\section{Mr. $\mathrm{XYZ}$ is to keep under observation for the next 48 hours.}

\section{His $\underline{B P}$ and Glucose levels are $\underline{\text { HIGH}}$}

Fig.5. Monitoring page.

Fig.5. is the web page displayed by the embedded web server which finds that the blood pressure parameter has exceeded the set limit and the symptoms of the cardiac arrest has occurred. 


\section{CONCLUSION}

By the survey and results obtained from the implementation of the system, we found that this type of extendable embedded web servers can be used in medical applications and devices with ease. This will make the job of doctors and nursing staff simple. It is very easy to understand the working functions, the configuration, and administration of these embedded servers. By using such embedded web servers, doctors can keep observing his patients from remotely. Such embedded web server occupies very small footprint of $400 \mathrm{~KB}$ only. But the web-enabled nature of this system may lead to cost effectiveness.

\section{REFERENCES}

[1] S. Vasudevan et al., 'Secure Telemedicine System for home health care', in West Virgina University, pp. 3940 .

[2] Andreas Ekstrand, Jones Ludvigsson, 'Generic web server in Embedded control Systems", in Department of Automatic Control, Lund Institute of Technology', ISSN, pp. 0280-5316.

[3] Miguel Domingues, "A Simple Architecture for Embedded Web Servers", by Universidade do Minho, Portugal, ICCA 2003, pp.113-120.

[4] I. Klimchynski. "Extensible embedded web server for internet-based data acquisition and control", in Proceedings of IEEE Sensors, Vol. 6, Issue: 3, 2006, pp. $804-811$.

[5] Bill McCombia, "Embedded Web Servers Now and in the Future", in Real Time Magazine, no.1 March 1998 pp.82-83.

[6] Klaus D McDonald-Maier, "The Embedded Web", in The Eighth Java \& the Internet in the Computing Curriculum Conference (JICC 8) Programme London Metropolitan University, Monday 26th January 2004.

[7] (Book) Bentham.J: "TCP / IP Lean - Web Servers for Embedded Systems", Second Edition, CMP Books, 560 pages, April 2002.

[8] American Heart Association's Heart Attack, stroke and Cardiac Arrest Warning Signs, Available: http://www.heart.org/HEARTORG/Conditions/HeartAtta ck/WarningSignsofaHeartAttack/Warning-Signs-of-aHeart-Attack_UCM_002039_Article.jsp\#

[9] Agranat Systems, Emweb, http://www.emweb.com

[10] AllegroSoft, RomPager, Available: http://www.allegro soft.com

[11] I.Agranat, "Embedded Web Servers in Network Devices", in Communication Systems Design, March 1998, pp. 30-36.
[12] Spyglass, Microserver, Available: http://www.spyglass.com

[13] Tao Lin, Hai Zhao, Jiyong Wang, Guangjie Han, Jindong Wang, "An Embedded Web Server for Equipments," in 2004 International Symposium on Parallel Architectures, Algorithms and Networks (ISPAN'04), 2004, pp 345350

[14] Janne Riihijarvi, Petri Mahonen, Mika J. Saaranen, Jussi Roivainen and Juha-Pekka Soininen, "Providing Network Connectivity for Small Appliances: A Functionally Minimized Embedded Web Server" in IEEE Communications Magazine, Vol. 39, Issue: 10 ,October 2001, pp. 74-79.

[15] "The Journal of American Medical Association", Vol.295 No.1 (Available: http://jama.amaassn.org/content/295/1.toc), January 2006.

[16] A.Maheswari, and V.Ramachandran, System for Detection of Vital Signals with an Embedded System", in African Journal of Information and Communication Technology, Vol. 6, No. 1, September 2011

[17] S. Vasudevan, K.J. Cleetus, "Low Cost Telemedicine for Home Health Care," wetice, pp.39, in Tenth IEEE International Workshops on Enabling Technologies: Infrastructure for Collaborative Enterprises, 2001.

[18] http://www.gwmicros.com/public_html/pages/products/i products/Embedded_Networks/Allegro/Embedded_Netw ork_Rom

[19] Technical Report "Secure Collaboration Technology for Rural Clinical Telemedicine" submitted to The National Library of Medicine, West Virginia University, 2009.

\section{AUTHORS PROFILE}

Chiranji Lal Chowdhary is an Assistant Professor (Sr.) and an internal part time research scholar at VIT University, Vellore. His research interests are in Image Processing and Computer Vision. He is a life member of ISCA.

Shynu P. G. is working as Assistant Professor and a research scholar in the School of Information Technology and Engineering, VIT University, Vellore, India. His area of interest includes Web services and internet computing. He is a member of Computer Society of India. 